CAHIERS DE

NARRATOLOGIE

\section{Cahiers de Narratologie}

Analyse et théorie narratives

31 Bis | 2017

Espace du récit, récit de l'espace en contexte germanique

\title{
Sur le seuil ? Hypothèses sur la spatialité dans la dramaturgie narrative de Roland Schimmelpfennig
}

\section{Kerstin Hausbei}

\section{(2) OpenEdition}

\section{Journals}

Electronic version

URL: http://journals.openedition.org/narratologie/7743

DOI: 10.4000/narratologie. 7743

ISSN: 1765-307X

Publisher

LIRCES

Electronic reference

Kerstin Hausbei, « Sur le seuil ? Hypothèses sur la spatialité dans la dramaturgie narrative de Roland Schimmelpfennig », Cahiers de Narratologie [Online], 31 Bis | 2017, Online since 26 June 2017, connection on 02 May 2019. URL : http://journals.openedition.org/narratologie/7743 ; DOI : 10.4000/ narratologie. 7743

This text was automatically generated on 2 May 2019.

Article L.111-1 du Code de la propriété intellectuelle. 


\title{
Sur le seuil ? Hypothèses sur la spatialité dans la dramaturgie narrative de Roland Schimmelpfennig
}

\author{
Kerstin Hausbei
}

Face au constat que les «nouveaux textes de théâtre ne peuvent plus être classifiés en vertu de spécificités typologiques du drame, ni par le respect de structures dialoguées ni par d'autres éléments de forme $»^{1}$, Theresia Birkenhauer propose de résoudre ce problème de classification en se demandant « dans quelle mesure les textes travaillent avec le potentiel des structures de représentation [Darstellungsstrukturen] du théâtre $»^{2}$. Traiter, comme je m'apprête à le faire pour Roland Schimmelpfennig, d'un auteur dramatique dans le cadre d'une réflexion liant espace et narration, suppose dans cette perspective de considérer le théâtre comme un art à la fois narratif et spatial et d'interroger le lien qu'établit l'auteur entre ces deux catégories au sein de sa dramaturgie. Les deux présupposés, celui de la spatialité comme celui de la narrativité du théâtre, ont été chacun source de vives polémiques qui ont, notamment en ce qui concerne la narrativité, mené à une redéfinition permanente des concepts. Ils peuvent être considérés comme deux modèles alternatifs formant sinon une opposition stricte, du moins un champ de tension dont je dessinerai les contours dans la première partie de mon article. Je développerai ensuite quelques hypothèses sur la façon dont Roland Schimmelpfennig (1967), qui s'est consacré à l'écriture après avoir été formé à la mise en scène et avoir pratiqué cette dernière ${ }^{3}$, qui écrit presque exclusivement pour le théâtre ${ }^{4}$ et compte parmi les auteurs dramatiques les plus joués, traduits et primés de sa génération, exploite ce champ de tension au sein d'une dramaturgie qu'il appelle luimême « narrative $»^{5}$. 


\section{Le théâtre comme art de l'espace}

2 Lorsque Oskar Schlemmer proclame en 1927 que « l'art de la scène est un art de l'espace » ${ }^{6}$, il réagit à une évolution rendue possible entre autres par les nouvelles techniques d'éclairage qui avaient au XIXe siècle remplacé les bougies: l'architecture scénographique pouvant se substituer désormais aux toiles de fond et au système des coulisses baroques, le théâtre put se spatialiser et libérer du même coup les mouvements des acteurs. Mais il s'attaqua également de façon polémique au primat du texte dramatique tel qu'il avait été instauré progressivement par la littérarisation du théâtre depuis l'époque des Lumières. Ranger le théâtre du côté des arts de l'espace signifiait, à l'époque de la (re)théâtralisation portée par les mouvements d'avant-garde, l'opposer à et le libérer de la littérature (dramatique) que Lessing avait classée parmi les arts du temps en raison de la linéarité de sa réception. La nature spatiale du théâtre, si évidente qu'elle soit (le théâtre, même dans sa forme dramatique, a toujours besoin d'un espace pour se réaliser), est donc utilisée dès cette époque très explicitement comme argument contre son caractère narratif, si l'on entend par là le fait que la représentation théâtrale réalise une suite d'événements dotée d'un sens, autrement dit une histoire.

Du côté de la théorie (et de la pratique) théâtrale, cette définition spatiale du théâtre est restée très influente. La formule de Schlemmer est reprise aussitôt par Max Herrmann dans son manifeste «Das Theatralische Raumerlebnis » ( "L'expérience spatiale au théâtre » ${ }^{7}$ ) pour légitimer la création des études théâtrales comme discipline autonome à côté de celle des études littéraires. Elle forme également un argument de choix dans la conception d'un théâtre " postdramatique » que Hans-Thies Lehmann distingue en 1999 du théâtre dramatique par une utilisation non narrative de la matérialité scénique ${ }^{8}$. Et elle sous-tend enfin, de façon moins polémique, les définitions minimales du théâtre des années 60. Peter Brook ouvre ses Ecrits sur le théâtre (ainsi le sous-titre de son ouvrage L'Espace vide issu d'une série de conférences données en 1965) par cette idée : «Je peux prendre n'importe quel espace vide et l'appeler une scène. Quelqu'un traverse cet espace vide pendant que quelqu'un d'autre l'observe, et c'est suffisant pour que l'acte théâtral soit amorcé. $»^{9}$

4 Introduisant la triade espace - acteur - spectateur, il varie la triade acteur - personnage représenté - spectateur sur laquelle Eric Bentley s'était appuyé un an plus tôt: «A impersonates $B$ while $C$ looks on $»^{10}$. Bentley avait ainsi maintenu l'idée d'une représentation et admis, du moins de façon implicite, la présence d'un support dramatique ${ }^{11}$. Mais au lieu d'accentuer la temporalité de l'action, il avait déjà basé sa définition sur une pratique culturelle collective représentée par deux activités simultanées et interdépendantes (incarner, regarder) située dans la triple spatialité caractéristique du théâtre : 1) l'ici et maintenant de l'espace théâtral qui comprend $\mathrm{A}$ et $\mathrm{C}$ « définissant entre eux un certain rapport $»^{12}$;2) l'espace scénique dédié à l'activité de $\mathrm{A}$; 3) l'espace dramatique où évolue $\mathrm{C}$, «une abstraction [qui] comprend non seulement les signes de la représentation, mais toute la spatialité virtuelle du texte, y compris ce qui est prévu comme hors-scène $»^{13}$ et ne se réalise que dans la réception du spectateur. La définition de Brook, laissant de côté la situation de représentation au profit de celle d'une simple performance, s'avère être plus ouverte dans la mesure où elle peut s'appliquer, à côté du théâtre dramatique (basé sur l'illusion), au théâtre épique (qui utilise l'acteur et la scène comme instances de médiation) ainsi qu'au théâtre postdramatique (qui fait disparaître la référentialité du 
signifiant derrière sa matérialité). En effet, ce n'est pas le texte qui est pensé ici comme préalable du spectacle, mais un acte fondateur : le prélèvement, dans un espace donné, d'un segment dont la nature sera transformée par une parole performative implicite ( «je peux [...] l'appeler une scène ») et la pratique culturelle collective qui en résulte. Séparé de l'espace environnant dans lequel se trouve le spectateur, l'espace scénique est "cadré », entouré d'une frontière (in)visible qui crée un effet de seuil comme l'explique Brook à partir de sa pratique :

Nous utilisons souvent comme espace de répétition un tapis, avec une convention très claire pour les acteurs : hors du tapis, on est dans la vie quotidienne, on peut faire ce que l'on veut [...], mais dès que l'on se trouve sur le tapis, il y a l'obligation d'avoir une intention claire, je dirais plus, d'être dans une vie intense ${ }^{14}$

$5 \mathrm{Au}$ fondement du théâtre dans sa définition spatiale se situe donc la création de ce que Michel Foucault appelle une « hétérotopie » ${ }^{15}$.Foucault introduit explicitement le théâtre comme exemple pour expliquer le « pouvoir de juxtaposer en un seul lieu réel plusieurs espaces, plusieurs emplacements qui sont en eux-mêmes incompatibles $»^{16}$, caractéristique des hétérotopies. Toutefois, pour Brook, l'hétérotopie ne réside pas dans le lieu théâtre qui accueille successivement différents spectacles, mais bien dans l'espace théâtral ${ }^{17}$ qui est créé dans chaque représentation/ performance, comme le souligne également Max Herrmann. Herrmann continue certes de se référer au paradigme d'un «théâtre représentationnel qui raconte une fiction » (Patrice Pavis), mais il propose une définition spatiale de l'événement théâtral. Sans renoncer à la dynamique transitoire (et donc temporelle) de l'action dramatique, il prétend que l'expérience esthétique du spectateur ne dépend pas de celle-ci, mais bien de la situation théâtrale elle-même: «L'espace que vise le théâtre est [...] un espace esthétique [Kunstraum] qui n'est créé que par une transformation intérieure plus ou moins importante de l'espace réel ; c'est une expérience vécue [Erlebnis], lors de laquelle l'espace scénique est transformé en un espace d'une autre nature. $»^{18} L^{\prime}$ « espace autre» que produit le théâtre peut donc selon Herrmann être identifié à l'émergence paradoxale d'un espace virtuel, mais réellement perçu, dans un espace réel dont il se distingue. D'un point de vue sémiotique, Patrice Pavis explique ce phénomène comme étant une illusion référentielle : «Le théâtre, du moins dans sa tradition mimétique (représentationnelle) pourrait se définir non seulement comme une mise en signe de la réalité, mais comme une réalité scénique que le spectateur transforme sans cesse en signe de quelque chose (processus de sémiotisation). $»^{19}$ Toutefois, pour Herrmann, ce processus n'est pas en premier lieu un décodage de signes renvoyant à l'espace dramatique, mais une expérience vécue (Erlebnis) au sens phénoménologique du terme :

L'activité créatrice, la participation créative du public à tout jeu dramatique réside en dernière instance dans le fait que le spectateur revit secrètement, qu'il recrée comme une ombre l'activité de l'acteur, dans un processus de réception qui passe moins par la vue que par un sentiment corporel, une pulsion secrète à exercer les mêmes mouvements, à produire dans son propre gosier le même son de la voix ${ }^{20}$.

6 C'est de cette façon que le spectateur participe selon Herrmann à la transformation de l'espace initiée par le travail de l'acteur :

Chaque être humain dépend dans la totalité de son habitus de l'espace dans lequel il se trouve : notre démarche, nos gestes, notre parole sont différents à l'air libre ou dans un espace clos et sont déterminés de façon décisive par les particularités de cet espace [...] le grand acteur crée lui-même son espace ou, plus précisément, il réinterprète l'espace scénique de telle façon qu'il devient pour lui un espace réel 
qui n'existe certes pas dans la réalité, mais qui détermine tout son habitus dans le

sens évoqué précédemment ${ }^{21}$.

Que l'expérience du basculement dans l'« espace autre » se fasse en lien avec une fiction dramatique ou non est donc finalement sans importance puisque la définition spatiale du théâtre est dans son essence performative : elle positionne celui-ci comme une pratique culturelle qui permet au spectateur (selon Herrmann à certains spectateurs dans certaines conditions) de franchir - avec les acteurs - la frontière qui sépare deux espaces simultanés, mais incompatibles entre eux.

\section{Le théâtre comme art narratif}

Du côté de la narratologie, c'est en raison même de la performativité sur laquelle repose la définition spatiale du théâtre que celui-ci a été considéré comme étant un art créant un rapport d'immédiateté avec son spectateur. Sur la base du couple d'opposition mimesis / diegesis, autrement dit de la régulation de la distance entre le pôle de réception et le monde fictif, le théâtre, y compris le «théâtre représentationnel qui raconte une fiction » (Patrice Pavis), a été classé par la narratologie comme étant non-narratif et le drame - texte écrit pour être représenté - a donc longtemps occupé «la position de l'Autre non narratif de la narratologie » (Monika Fludernik ${ }^{22}$ ). C'est là un « paradoxe quasi fondateur de la narratologie » (Benoît Hennaut ${ }^{23}$ ) si l'on considère que la narratologie littéraire du XXe siècle s'appuie sur Platon et Aristote chez qui l'opposition mimesis/diegesis n'apparaît pas sous cette forme ${ }^{24}$. Aussi, au sein de la narratologie récente, transgénérique et transmédiale, les voix ne manquent pas qui interrogent de façon critique les critères de cette exclusion et réclament la révision de ce verdict ${ }^{25}$. Parallèlement, en réaction à la thèse d'un théatre postdramatique non-narratif (Lehmann), la question du retour, de la persistance, voire de l'apparition de la narration sur les scènes de théâtre est fréquemment débattue aujourd'hui dans le milieu du théâtre ${ }^{26}$. Les lignes de partage entre narratif et non-narratif varient dans ces débats en fonction des critères retenus pour définir la narrativité.

9 La définition la plus large est celle qui, sur la base de la différenciation entre types de texte introduite par Chatman, fait de la narrativité une qualité de l'objet représenté - l'histoire -, qui s'oppose à des objets non narratifs (descriptifs, argumentatifs...) par la présence d'une séquence temporelle impliquant des événements, des personnages et des lieux reposant sur une sélection et un agencement ${ }^{27}$. Le drame et le théâtre mimétique représentationnel se situent dans ce cas clairement du côté de la narrativité. Le théâtre épique, s'il comporte des éléments narratifs, introduit également des éléments argumentatifs. Ceux-ci peuvent devenir dominants dans l'organisation spatiale, comme c'est par exemple le cas chez Piscator, mais la fonction argumentative dans son ensemble reste subordonnée à la fonction narrative dont elle constitue le commentaire, la contextualisation, et ${ }^{28}$. Selon ses théoriciens, le théâtre postdramatique serait, quant à lui, à opposer à ces variantes d'un «théâtre à fable » (« Fabeltheater " ${ }^{29}$ ), en raison de son abstraction $^{30}$, ainsi qu'en raison de sa structure parataxique qui rompt avec la logique de l'action et avec la communication d'une signification qui lui est inhérente :

L'art de la parataxe consiste à former cette juxtaposition de telle façon que son effet reste ouvert sur le plan de la signification, alors qu'il est actif sur le plan des émotions. Dans la suite d'événements de type dramatique, il existe entre la cognition [Erkennen] et l'effet émotionnel une relation dialectique. Dans la suite d'événements de type parataxique, le fil rouge d'une action qui guide la cognition 
manque. Le spectateur doit être prêt à tout moment à une participation sensorielle. C'est seulement s'il accepte et vit celle-ci que le déroulement des parataxes devient pour lui l'expérience d'une forme artistique. [...] Il devient l'auteur des événements qu'il a lui-même vécus. Mais il doit d'abord les avoir vécus et il ne doit pas se barrer le chemin vers cette expérience par une observation qui cherche une signification ${ }^{31}$.

\section{définition spatiale du théâtre.}

La deuxième définition de la narrativité repose sur le caractère médiatisé de la représentation de l'histoire. La ligne de partage dépend ici de l'identification des effets de cette médiation (de la présence du niveau du discours ou récit donc) et, éventuellement, de l'instance qui peut être tenue pour responsable de ceux-ci. Si la narratologie «classique » d'un Stanzel et Genette exclut sur cette base le drame et le théâtre de son champ, il est aujourd'hui souvent admis que le drame de forme fermée ${ }^{32}$ - et a fortiori celui de forme ouverte - repose sur une médiation et sur une distinction histoire / récit. Après la démonstration en 1956 par Peter Szondi de l'existence d'une instance épique dans le drame moderne, l'un des premiers a avoir intégré des concepts de la narratologie (dont celui de perspective) au modèle du texte dramatique est Martin Pfister [Das Drama, 1977]. Mais sur le plan terminologique, Pfister maintient l'idée d'un rapport non médiatisé ( unmittelbar ") entre le "système de communication interne " (axe scène-scène) et le «système de communication externe » (axe scène-salle). Pour évacuer cette contradiction, un procédé commode et souvent emprunté consiste à distinguer entre le texte du drame (aussi appelé script) et le drame représenté sur scène, afin de pouvoir identifier le didascale ${ }^{33}$ comme instance de médiation qui disparaîtrait à la représentation au profit d'un rapport non médiatisé avec le spectateur ${ }^{34}$. Le modèle narratologique de Wolf Schmid qui divise la narration en quatre opérations constitutives permet toutefois de montrer que l'intervention d'une instance de médiation (qui introduit aussi des effets de perspective) intervient dès la constitution de l'histoire et du récit et ce indépendamment de la représentation médiale choisie. Il permet donc de distinguer la médiation (qui se reflète dans la composition d'ensemble) de la médialisation / modalisation (qui n'en est qu'un des aspects ${ }^{35}$ ): Schmid distingue par analogie avec la rhétorique entre 1) le niveau des «événements » (inventio) qui comporte les personnages, les lieux et les événements au stade parataxique et infini de la chronique, 2) le niveau de l'« histoire » (dispositio dans un ordo naturalis) qui est une forme limitée (avec un début et une fin), créatrice de sens, qui provient de la sélection de certains événements et certaines particularités et de leur hiérarchisation, 3) le niveau du « récit » qui désigne la composition des éléments retenus dans un ordo artificialis sous la forme d'une linéarisation qui peut comporter des permutations et 4) le niveau de la «représentation du récit » (elocutio/lexis) qui correspond à sa présentation médiale (verbale, filmique, musicale, figurative, etc ${ }^{36}$.).

La fonction narratoriale contenue dans le texte dramatique au niveau de la conception de l'histoire et du récit reste bien entendu perceptible dans le passage à la scène (elle est souvent attribuée à un narrateur ou auteur impliqués ${ }^{37}$ ) et on peut la distinguer de celle opérée par le théâtre lui-même. Holger Korthals, pourtant lui-même adepte d'une séparation entre le texte dramatique, symbolisé et médiatisé, et la représentation, iconique et immédiate, concède à ce sujet que le «spectateur illusionné qui passe outre la médiation de la scène, des acteurs et de l'appareil théâtral et participe en quelque sorte directement à l'action peut être tenu à son tour pour une illusion de certains théoriciens $\mathrm{du}$ drame $\aleph^{38}$. On peut ajouter à cela que toutes les esthétiques non naturalistes 
s'appliquent à rendre visible le théâtre dans son rôle de médiation ${ }^{39}$. Mais même en dehors de toute considération esthétique particulière, Hans-Peter Bayerdörfer souligne la fonction narrative inhérente au jeu d'acteur lui-même en raison de sa double nature - mimétique et diégétique: "L'acteur agit en tant que participant à l'action interpersonnelle [interpersonales Geschehen], mais de façon latente, corporelle, toujours également comme médiateur de l'action, c'est-à-dire comme interprète-narrateur corporel de sa propre incarnation. $»^{40}$

13 La définition la plus étroite de la narrativité réclame une situation manifeste de narration verbale et donc la présence d'une instance narrative incarnée. Ces situations ont bien sûr toujours existé dans le drame et le théâtre, sont bien connues et souvent répertoriées dans la narratologie transgénérique ${ }^{41}$. Du point de vue de l'utilisation de l'espace scénique, on peut distinguer deux situations différentes :

1. les situations dans lesquelles l'acte de narrer fait ponctuellement partie de l'espace dramatique et reste séparé ontologiquement de l'espace dans lequel se situe le spectateur : le récit, pris en charge par un personnage, peut alors soit thématiser un horsscène et/ou hors-temps et se situer dans la même sphère ontologique que les personnages (récit de messager, teichoscopie, etc.) soit être tenu pour fictif par les personnages eux-mêmes et constituer une sphère ontologique propre (conte, chant, parabole, etc.);

2. les situations dans lesquelles l'acte de narration crée une rupture ontologique avec l'espace dramatique et se situe - en apparence - dans une sphère commune avec le spectateur comme c'est le cas lorsque l'acte de narration se situe dans une sphère qui dispose d'un personnel propre (p.e. présentateur générateur) ou dans le cas d'une métalepse, lorsque certains personnages traversent la frontière ontologique, rompant ainsi le pacte fictionnel (p.e. l'a parte, les adresses au public de certains personnages brechtiens ou des épilogues dans la tradition de Shakespeare). Mais comme il ne s'agit pas d'une parole spontanée de l'acteur qui quitterait effectivement son rôle pour s'adresser directement au spectateur dans une situation authentique de communication, cette sphère correspond bien sûr au niveau extradiégétique d'un narrateur hétérodiégétique ou homodiégétique dans le roman.

Une variante de cette deuxième forme joue un rôle non négligeable dans certaines pratiques scéniques "postdramatiques $»^{42}$ où les acteurs endossent, souvent dans une parole monologique, pour la durée d'une simple anecdote ou de tout un spectacle, le rôle d'un «narr-acteur» (Simone Soriani) dans une interaction directe avec le public, en apparence sans passer par le détour de l'incarnation d'un personnage ${ }^{43}$. L'acte de narrer dans sa fonction anthropologique de structurer et de communiquer des expériences humaines - dont Walter Benjamin avait pronostiqué la crise après la première guerre mondiale ${ }^{44}$ et sur laquelle insistent toutes les conceptions de la narrativité factuelle - se trouve alors exposé sur la scène d'un théâtre qui refuse de jouer lui-même ce rôle structurant pour le spectateur ou qui délègue cette fonction au spectateur ${ }^{45}$.

On peut penser que les écritures dramatiques actuelles se sont inspirées à la fois du théâtre épique, de pratiques du théâtre postdramatique, mais aussi de certaines pratiques de mise en scène ou encore de dramatisations de romans pour proposer un théatre narratif d'un nouveau type dans lequel on constate l'arrivée massive de parties narrées au sein même du texte primaire destiné à être prononcé sur scène par les acteurs ${ }^{46}$. Des pans entiers - voire l'histoire dans son ensemble - peuvent être confiés à des instances narratives de type «narr-acteur » qui prennent en charge, parfois de façon chorale, une 
diégèse qui ne se concrétise plus de façon mimétique sur scène. Narratif au sens étroit du terme, ce théâtre peut parallèlement, notamment dans sa version chorale, développer un fort potentiel performatif lié à des effets de présence, de volume et de masse sonore, etc. Il tend à imposer le langage dans sa matérialité autant que dans sa fonction sémantique / narrative.

Une autre variante, moins exclusive, plus brechtienne, de cette nouvelle narrativité consiste à faire alterner l'acteur dans la prise en charge de son rôle entre le mode mimétique et le mode diégétique. Mais à la différence des pratiques antérieures, ces récits à la première ou à la troisième personne ne sont souvent ni intégrés à une situation dramatique (récit de messager, teichoscopie, etc.) ni confiés à une instance narrative clairement identifiée comme telle et dépassant la subjectivité d'un personnage (choeur, narrateur) ni même introduits par des ruptures aptes à signaler au spectateur le changement de niveau narratif (théâtre épique). On voit ainsi apparaître à côté du théâtre épique de type brechtien ou piscatorien - reposant sur une séparation, y compris spatiale, entre un niveau diégétique (réservé à la fonction narrative) et un niveau extradiégétique (réservé à son exégèse) - un théâtre narratif "postépique » (Bernd Stegemann ${ }^{47}$ ), dans lequel la fonction narrative elle-même fait l'objet d'une hybridation, d'un basculement, voire d'un glissement imperceptible entre mode diégétique et mode mimétique ${ }^{48}$. Cette nouvelle instabilité des niveaux narratifs implique également un nouveau rapport - plus instable - entre l'espace scénique et l'espace dramatique et modifie considérablement l'expérience esthétique du spectateur dans l'espace théâtral puisqu'il est invité à réajuster à tout moment sa distance par rapport au monde représenté, voire à la choisir. Cette variante d'un théâtre narratif crée donc un pont vers la définition spatiale du théâtre.

\section{La narrativité de la dramaturgie de Schimmelpfennig}

Entre narrativité et spatialité, Roland Schimmelpfennig semble avoir choisi son camp. Non seulement, il affirme dans son cours de poétique Ja und Nein en 2014 que le théâtre est narratif par nature - une affirmation qu'il étend pour sa part également au théâtre postdramatique ${ }^{49}-$, mais il évolue aussi dans sa dramaturgie vers des formes de narrativité de plus en plus radicales, passant d'une forme de narrativité au sens large du terme (théâtre à fable) dotée de dialogues, d'un didascale et d'un espace iconique (p.e. Aus den Städten in die Wälder, aus den Wäldern in die Städte, création 1998) à des formes de présentation, dans lesquelles la médiation est exhibée et le récit oralisé supplée de plus en plus au mode représentationnel et iconique. Apparaissent ainsi au tournant du millénaire des formes postépiques avec un statut hybride du texte primaire sans espace iconique (p.e. Une Nuit arabe), ainsi que des montages épiques où les dialogues sont interrompus régulièrement par des récits monologués ad spectatores dans lesquels les personnages se muent tour à tour en exégètes subjectifs des parties dialoguées à la manière du «confessionnal » de la télé-réalité ou livrent au spectateur, dans un esprit plus brechtien, des informations contextuelles sur eux-mêmes et leurs habitudes de vie (p.e. Push up). Dans cette phase, Schimmelpfennig exploite également les possibilités d'anachronie offertes par la technique du montage pour introduire de forts effets de perspective (p.e. en revenant plusieurs fois sur une même scène en la faisant présenter par différents personnages impliqués dans Avant / Après ou en montrant la même scène deux fois en la faisant commencer un peu plus tôt la seconde fois dans La Femme d'avant). 
A partir de 2008, Schimmelpfennig franchit une étape supplémentaire en créant des pièces entièrement narrées dans lesquelles il abolit l'unité acteur / personnage au profit d'un choeur de narr-acteurs qui prennent en charge dans des constellations changeantes (en respectant toutefois la répartition hommes/femmes) aussi bien le récit d'action que les rares dialogues, introduits systématiquement par des verbes du dire (p.e. Idomedeus).

Sans pour autant abandonner les formes utilisées précédemment, Schimmelpfennig élargit ainsi pendant dix ans continuellement sa palette de formes dramaturgiques narratives et couvre désormais à lui seul tout le spectre des dramaturgies narratives évoqué plus haut : il est un «mécanicien de précision de la narration » (Franz Wille $\left.{ }^{50}\right)$.

Nous avons vu plus haut que définir le théâtre comme art de l'espace ou comme art narratif équivaut à accentuer respectivement son caractère performatif ou médial, à le faire basculer du côté de la phénoménologie ou de la sémiotique. C'est donc aussi et avant tout définir différemment la place et l'activité du spectateur, même si ces modes de réception ne sont bien sûr pas exclusifs l'un de l'autre. La perception du spectateur oscille toujours, tantôt de façon volontaire, tantôt de façon involontaire, entre médialité, matérialité, sémioticité et esthéticité du spectacle ${ }^{51}$. Mais un texte qui travaille « avec le potentiel des structures de représentation [Darstellungsstrukturen] du théâtre ", pour reprendre la formulation de Theresia Birkenhauer de laquelle était partie notre réflexion, devrait contenir des mécanismes dramaturgiques qui visent, favorisent et guident cette oscillation pour créer une tension productive entre l'aspect narratif et l'aspect spatial du théâtre. Il va de soi que les mises en scène apporteront leurs propres accentuations à ce sujet. Aussi, tout comme la fonction narrative des lieux dramatiques ${ }^{52}$, nous les laisserons de côté dans les hypothèses qui suivent. Celles-ci (nécessairement fragmentaires en raison de la richesse des formes employées) visent exclusivement à sonder l'utilisation que fait Schimmelpfennig du potentiel spatial du théâtre dans sa dramaturgie fortement ancrée dans la narrativité. Nous nous intéresserons donc successivement au trois formes de spatialité développées plus haut: la parataxe, l'hétérotopie comme qualité du lieu théâtral et l'hétérotopie comme propriété de l'espace théâtral.

\section{La parataxe comme seuil entre spatialité et narrativité}

21 Avec Vor langer Zeit im Mai, sa seule incursion dans le domaine postdramatique créée en 2000, Schimmelpfennig livre une pièce non narrative dans laquelle le principe de la parataxe est intégré de deux façons distinctes. D'une part, il crée sur l'axe diachronique, à partir d'un nombre très limité de motifs, une suite de courtes séquences dans lesquelles ces motifs sont combinés et variés sur un mode fragmentaire, répétitif et obsessionnel sans qu'une évolution depuis une situation de départ vers une situation d'arrivée soit perceptible. D'autre part, sur l'axe synchronique, il fait coexister deux sphères sans contact direct et sans lien sémantique évident entre elles. Elles sont construites, sur le plan formel, de façon complémentaire: l'une, privée de parole (à l'exception d'une chanson), est entièrement constituée de micro-actions isolées décrites dans des didascalies, l'autre, réservée exclusivement à un dialogue fragmentaire, est privée de didascale (au point que les voix « Elle » et « Lui » qui la constituent n'apparaissent même pas dans la liste des personnages). En contrepoint à cette construction doublement parataxique, Schimmelpfennig suscite chez le spectateur une attente de narrativité qu'il ne satisfait pas. 

de la pièce ( « Il y a longtemps au mois de mai »). Elle est écrite à la manière d'un chant populaire, navigue d'une sphère à l'autre ${ }^{53}$ et évoque de façon allusive le souvenir d'une amitié passée (ou s'agit-il d'un amour ?). Elle introduit ainsi un noyau de narrativité qui reste réduit à son strict minimum : une temporalité linéaire qui sépare deux situations (être à deux, être seul). Aucun détail n'est donné ni au sujet des protagonistes, des lieux et modalités ou même de la nature de la relation, ni au sujet des causes, de la modalité ou de la temporalité de sa fin. Plutôt que de déboucher sur une narration, la chanson invite ainsi à une lecture allégorique sur le thème de la finitude de la vie humaine en contraste avec le temps circulaire de la nature (cercle des saisons, renouvellement des générations). dialogue entre « ELLE » et «LUI ». Le topos d'un passé commun est introduit sur un mode allusif, à travers des objets (un vélo, une valise) et des personnes (une amie pour chacun ; une femme qu'ils ont entendu chanter) au sujet desquels se questionnent mutuellement les voix. S'il peut supposer qu'ils soient chargés d'une histoire pour les voix, ces éléments n'en restent pas moins pour le spectateur à l'état de noms communs sans caractéristiques propres. Débouchant systématiquement soit sur des échanges purement phatiques ${ }^{54}$, soit sur des ripostes ${ }^{55}$, soit sur des esquives ${ }^{56}$, les échanges constituent certes, chacun pris individuellement, des noyaux de narrativité composés de la combinaison des figures de l'échange verbal (mouvement vers + mouvement vers, mouvement vers + riposte, mouvement vers + esquive), mais n'aboutissent ni à un récit d'événements passés, ni à un changement de la situation présente qui pourrait former un événement dans l'univers dramatique. Le dialogue tend ainsi à exposer les actes de langage pour eux-mêmes : ils deviennent des matériaux dans un arrangement abstrait qui, à défaut de construire une fable située dans un espace dramatique, reste cantonné à l'ici et maintenant du spectacle, dans une durée non narrative.

Il rejoint en cela la sphère visuelle qui combine et varie les mêmes motifs, tout en les démultipliant (plusieurs hommes à vélo et femmes à la valise) et en les enrichissant d'éléments concrets qui ne sont pas évoqués dans le dialogue (les hommes à vélo tournent en rond, prennent de l'élan et s'écrasent contre le mur ; les femmes à la valise, souvent à moitié nues, chutent ou entrent en collision avec des couples amoureux). Elle fait intervenir également des personnages qui n'apparaissent pas en tant que tel dans le dialogue. Ceux-ci s'engagent dans des micro-actions sérielles sur le mode de la répétitionvariation: une femme en robe rococo fait des apparitions en passant par des portes dérobées (est-ce l'amie de l'un ou de l'autre ?), des couples amoureux s'embrassent et se font renverser (est-ce le couple qu'ils ont jadis formé ?), des femmes balaient le sol, parfois en chantant (une réminiscence de la femme qu'ils ont jadis entendu chanter ?).

Comme nous l'avions déjà observé pour la chanson, le blocage de narration peut mener à une réflexion sur la nature multiple du temps: le temps linéaire du changement (narrativité) se superpose au temps circulaire de l'éternel retour du même (répétitions incessantes des mêmes micro-événements) et débouche ainsi sur l'expérience d'une durée (ici et maintenant du spectacle). On retrouve le même type de superpositions sur le plan des motifs. Le vélo, image du mouvement linéaire, s'inscrit parallèlement dans la circularité des ronds. Le balai, image de l'effacement des traces, est associé à la durée du souvenir (chanson). La valise enfin sert à déplacer (changement) un contenu qui reste identique à lui-même (son passé ?) et l'inscrit dans une durée. 

reste dans l'indétermination (Wolfgang Iser). Il pourrait certes être narratif. La sphère visuelle renverrait alors sur un mode fantasmagorique et / ou burlesque à une phase antérieure (la première rencontre?) de ce couple d'amants (d'amis ?) qui se sont perdus de vue. Mais comme aucun indice ne permet de reconstituer la série d'événements qui ont mené de la situation antérieure (du reste entièrement hypothétique) à la situation présente (incertaine), la narration reste là encore bloquée. Le lien entre les deux sphères est pour cette raison d'abord et avant tout celui d'un parallélisme structurel puisque dans les deux sphères une tension est produite entre les éléments narratifs (récits minimaux) au niveau de la microstructure et le caractère parataxique de la macrostructure. Cette contradiction structurelle, qui interdit au spectateur de se projeter dans un espace dramatique et d'accorder à la scène le statut d'une instance de médiation, le renvoie à l'expérience de la matérialité des signes scéniques et l'expose in fine - par la frustration qui résulte de la non-satisfaction - à l'expérience de son propre désir de narration.

Dans beaucoup de pièces narratives de Schimmelpfennig, on retrouve cette tension entre une microstructure narrative et une macrostructure non narrative, mais elle ne produit pas ici le même effet. Schimmelpfennig l'introduit par le biais de ce que l'on appellerait dans le roman une «forme spatiale ", une organisation d'ensemble qui remplace le lien temporel et causal de l'action par une construction narrative basée sur la fragmentation, le montage d'éléments disparates et la juxtaposition de plusieurs histoires indépendantes ${ }^{57}$. Elle ressemble donc à la forme parataxique de Vor langer Zeit im Mai, mais travaille au niveau de la microstructure avec des noyaux narratifs beaucoup plus développés. Depuis Une Nuit arabe, le caractère spatial de cette forme est de surcroît à prendre à la lettre, puisque Schimmelpfennig crée désormais pour ce type de pièces un lieu fictif unique subdivisé en plusieurs espaces qui deviennent le cadre pour les histoires de plusieurs personnages ou couples de personnages (un hôtel dans Avant/Après; une rue de Berlin dans Auf der Greifswalder Straße ; un immeuble d'habitation dans Une Nuit arabe et Le Dragon d'or; un immeuble de bureau dans Push Up). A partir du tournant du millénaire, Schimmelpfennig renonce donc à la radicalité que revêt cette forme encore dans une pièce comme Keine Arbeit für die junge Frau im Frühlingskleid où le principe de l'indétermination, inhérent à la forme parataxique, constitue un frein à la sémiotisation des histoires de la microstructure, du lien entre les différentes histoires dans la macrostructure et de celui entre les deux sphères de l'espace dramatique (un espace autoréférentiel occupé par des personnages-comédiens installé sur la rampe devant le rideau fermé et un espace référentiel installé sur la scène à l'aide de décors peints), alors même que le personnage titre crée un lien formel entre tous ces éléments. Car la variante concrète de la forme spatiale (le lieu unique subdivisé) fait du principe parataxique une propriété du lieu dramatique. Que ce rapport métonymique, motivé par la nature du lieu, soit ou non doublé d'un contact direct entre les personnages de différentes histoires ou d'un lien d'analogie qui découle d'un motif commun est donc finalement sans importance puisqu'il apporte à lui seul un lien évident entre les différents noyaux de narrativité et fait basculer la parataxe du côté de la narrativité. Le seuil est donc ici franchi.

\section{Le théâtre comme lieu hétérotopique}

Dans les pièces narratives de Schimmelpfennig, les personnages sont souvent avant tout caractérisés par leur rapport spécifique à l'espace. Schimmelpfennig renonce la plupart 
du temps à la création d'un espace mimétique / iconique (que ce soit par l'indication d'un décor ou par l'indication de micro-actions) ou même l'interdit par le recours à de forts effets de distanciation (notamment l'abolition de l'unité acteur / personnages dans les pièces entièrement narrées, ainsi que dans Le Dragon d'or, où Schimmelpfennig fait apparaitre dans la liste des personnages des profils d'acteurs caractérisés par leur sexe et leur âge qui prennent en charge plusieurs personnages d'un profil opposé au leur). A la place, en complément ou en concurrence avec l'espace mimétique, Schimmelpfennig recourt à une construction diégétique de l'espace portée par les personnages. Les personnages tantôt se réfèrent dans leur dialogue à l'espace et / ou en font un thème central dans leurs échanges, tantôt (dans les pièces narrées et postépiques) en donnent des descriptions et / ou le construisent en faisant le récit de micro-actions qui s'y déroulent. Pour paraphraser Herrmann, on pourrait dire que ces personnages créent chacun autour d'eux un espace de façon performative. Le spectateur est invité à se projeter mentalement dans cet espace, à le co-créer dans son imaginaire ou, du moins, à le dénoter à partir des informations dont il dispose. Schimmelpfennig se sert souvent de la forme spatiale en général et du lieu unique subdivisé en particulier pour créer plusieurs de ces espaces diégétiques qui coexistent, se suivent ou se superposent. Le théâtre déploie alors son potentiel hétérotopique dont le spectateur devient conscient lorsque des contradictions sont introduites.

Une variante simple de ce principe consiste à travailler avec des effets de cadrage qui aboutissent à des variations et superpositions d'échelles. Dans de nombreuses pièces, on trouve ainsi la coexistence dans un même lieu de l'espace mondialisé, vaste et ouvert, dans lequel vivent ou dont rêvent des personnages issus du management et commerce international, du transport aérien, du milieu des ONG, de la migration, etc. (« Vous avez terminé vos études en Amériques, à la suite de quoi vous avez travaillé pour deux sociétés différentes au Japon, en Corée et à Taïwan $»^{58}$ ) et d'un espace local, réduit et étriqué, dans lequel évoluent les gardiens d'immeubles, artisans, couples bourgeois, commerçants locaux, etc. («Le bâtiment est grand, vraiment grand, seize étages ${ }^{59}$ ). Cette distinction manichéenne est problématisée dans le système de communication externe, puisque Schimmelpfennig crée pour chacun de ces types de personnages une superposition paradoxale des deux échelles. Les hôtesses de l'air dans Le Dragon d'or parcourent certes le monde ( « les deux ont un long vol derrière elles, elles viennent de Santiago du Chili, c'est presque au bout du monde vers le sud $\left.»^{60}\right)$, mais la cabine de l'avion, un «non-lieu » (Marc Augé) extrêmement restreint, les enferme et les isole à la fois du monde qu'elles survolent et des personnes qui y vivent ou périssent (comme le fait ressortir le dédoublement paradoxal de l'espace lorsque l'une des deux perçoit des boat people alors que l'autre lui oppose que l'avion vole à une altitude de dix mille kilomètres). Et la même double logique caractérise aussi la cuisine exiguë où est enfermé le jeune Chinois sans papiers dans la même pièce, elle aussi un non-lieu mondialisé, comme le fait ressortir non seulement la localisation paradoxale "thaï-chinois-vietnamien» qui accompagne systématiquement la mention du « resto rapide», mais également le fait qu'en l'absence de statut légal, l'immigré clandestin reste isolé de la société environnante et de son système de soins, condamné à mourir d'une septicémie après s'être fait arraché sa dent malade par ses compatriotes. La mobilité géographique des personnages ne les mène donc pas ailleurs, tout comme la proximité géographique (entre la salle et la cuisine du restaurant ou entre la loge et les bureaux de l'immeuble) ne les rapproche pas. Ils restent dans le huis clos de leur « emplacement » (Michel Foucault), une logique qui est soulignée 
également par le fait que la discussion des deux hôtesses de l'air se situe en quelque sorte simultanément dans la salle du restaurant où le dialogue est d'abord installé sur le mode diégétique (à deux pas donc de la cuisine dans laquelle périt le jeune Chinois dont une des hôtesses trouvera la dent dans sa soupe) et dans la cabine de l'avion en plein vol, situation à laquelle se réfèrent les hôtesses dans leur dialogue mimétique. Pour le spectateur, l'espace devient ici à la fois uniforme malgré son apparente hétérogénéité et hétérotopique malgré cette uniformité puisque chaque personnage participe au même moment à des espaces différents dont la nature est contradictoire (espace social clos et espace géopolitique ouvert dans les exemples précédents). Dans Avant/Après, Schimmelpfennig distord le décalage entre cadrages divergents jusqu'au microcosme de la biologie et, surtout, au macrocosme de l'astronomie, aboutissant ainsi à la collision entre un espace "vécu» et un espace "su " qui glisse à son tour vers un statut ontologique différent lorsque Schimmelpfennig introduit un organisme dangereux venu de l'espace et un insecte aux propriétés fantastiques.

Pour le spectateur, l'espace devient ainsi une donnée relative. Schimmelpfennig rompt en cela avec le concept d'un espace-contenant, s'intéresse à des logiques spatiales (analogues aux logiques temporelles dans Vor langer Zeit im Mai) et se rapproche ainsi des positions qui sont débattues à partir du début du XXe siècle dans la philosophie et les sciences sociales et que nous avons vues à l'oeuvre dans la définition spatiale du théâtre. Dans cette optique, l'ordre sémantique est conçu comme pré-existant à l'expérience de l'espace. Pour le formuler avec Ernst Cassirer, « l'espace ne reçoit son contenu déterminé et son agencement particulier que de l'ordre du sens au sein duquel à chaque fois il se configure. » Cassirer identifie trois grands ordres, l'ordre mythique, l'ordre esthétique et l'ordre théorique, et stipule qu'en fonction de l'ordre activé «la 'forme' de l'espace elle aussi change $»^{61}$. On retrouve cette idée chez Foucault qui - basculant l'espace esthétique dans les hétérotopies - introduit une classification légèrement divergente. Son espace de localisation correspond à l'ordre mythique de Cassirer, une topologie qui sépare sacré et profane, accessibilité et inaccessibilité, familiarité et étrangeté, promesse de bonheur et danger menaçant et dans laquelle "[t]out lieu et toute direction a [...] une qualité mythique déterminée et en ce sens est en quelque sorte chargé ${ }^{62}$. L'étendue de Foucault correspond à l'ordre théorique de Cassirer. L'emplacement, une catégorie absente du système de Cassirer, est un ordre préoccupé par le " problème de savoir quelles relations de voisinage, quel type de stockage, de circulation, de repérage, de classement des éléments humains doivent être retenus de préférence dans telle ou telle situation pour venir à telle ou telle fin $"^{63}$. Foucault fait coïncider son modèle avec celui d'une succession historique (moyen-âge, temps modernes, époque contemporaine), mettant au jour ainsi une temporalité de l'espace qu'avaient déjà thématisée les grands dramaturges des seuils entre deux chronotopes que sont Shakespeare et Tchekhov, tous deux des références importantes pour Schimmelpfennig.

31 Schimmelpfennig lui-même se détourne de ce modèle chronologique au profit d'une simultanéité de plusieurs logiques spatiales pouvant aller d'une simple confrontation d'emplacements incompatibles jusqu'à la coexistence paradoxale d'ordres spatiaux au sens de Cassirer et Foucault comme c'est le cas de façon très explicite encore dans une pièce précoce comme Aus den Städten in die Wälder, aus den Wäldern in die Städte. La ville y est conçue comme un espace de l'emplacement, alors que la forêt constitue une espace mythique où, comme l'explique le bûcheron, on se transforme en arbre lorsqu'on quitte les sentiers et on ne doit pas abattre des arbres pour des finalités dépourvues de nécessité 
(parmi lesquelles figure pour lui le «jeu» de scène) puisque la forêt « hait » quiconque "prend aux arbres leur habitat sans la moindre raison " ${ }^{64}$. Cette simultanéité des ordres spatiaux permet également le basculement provisoire ou définitif d'un ou de plusieurs personnage(s) dans un autre ordre spatial. Ce mouvement qui forme un contraste avec l'immobilité d'autres personnages et / ou avec l'immobilité socio-culturelle évoquée plus haut forme le sujet des pièces au sens que Youri Lotman a introduit dans la narratologie ${ }^{65}$. Au basculement de l'« emplacement » vers l'« espace théorique » s'ajoute le basculement, fréquent chez Schimmelpfennig, dans l'« espace mythique » où les personnages se font engloutir dans un monde parallèle au statut ontologique différent proche de l'univers du mythe et du conte. La plupart du temps, ce sont des situations liminales (le rêve et l'érotisme, la mort et l'art) qui constituent le seuil entre l'emplacement (ville, hôtel, immeuble d'habitation, etc.) et l'espace mythique ouvrant sur l'« espace autre ». Dans Une Nuit arabe, le corps nu de Franziska, pure présence et matérialité qui échappe à la logique de l'emplacement puisque la jeune femme comme tous les soirs ne se souvient plus ni de son identité ni de son métier, fait basculer les autres personnages dans l'espace mythique : ce corps les attire et les piège puisqu'à son contact, ils sont entraînés dans un orient fait d'enlèvements, de scheiks, de harems et d'hommes enfermés dans des bouteilles, dans lequel Franziska situe dans son rêve son propre passé et qui contraste singulièrement avec la vie sage et chronométrée que mènent les personnages arabes Fatima et Kalil au début de la pièce. Dans Le Dragon d'or, c'est en agonisant que le Chinois quitte l'espace de l'emplacement pour rejoindre l'espace mythique. Il peut alors entrer en contact avec sa famille à travers le trou laissé par la dent arrachée et après sa mort, son corps rentre en Chine, portée par l'eau de la rivière et de la mer, une variante du voyage mythique vers l'Autre monde. Dans Avant/Après, c'est en contemplant une peinture qu'un homme disparaît dans celle-ci et reste enfermé dans cet espace autre. Dans Aus den Städten in die Wälder, aus den Wäldern in die Städte, une pièce aux accents très shakespeariens, c'est le projet de la reconstruction du théâtre après un incendie qui attire les personnages de la ville dans l'espace mythique de la forêt. Ils cherchent «au plus profond de la forêt " " un bois particulier, unique et étrange ", impossible à trouver en ville, mais nécessaire pour la construction du plancher de scène. Ils se font piéger par l'espace mythique dont ils ignorent les règles et se transforment en arbres. Inversement, le bûcheron et le menuisier qui vivaient jusqu'ici dans la forêt peuvent quitter l'espace mythique à la fin de la pièce. C'est encore le théâtre qui motive ce changement. Car le menuisier a assisté dans sa jeunesse à une représentation théâtrale (Macbeth) et transmet à son entourage le récit de son expérience. Celle-ci correspond au rapport non médiatisé à l'univers dramatique (tel que le prévoit la narratologie " classique ») mais inscrit celuici dans le mode spatial de réception. Car le menuisier ne fait pas le récit de la fable, mais celui de sa propre expérience hétérotopique vécue avec la plus grande intensité sur un mode illusionniste. Ce récit procède par à-coups, passant d'une situation (et d'un décor) à un autre. Le menuisier se montre certes conscient de la théâtralité de son expérience ( $" A$ impersonates B while C looks on ») puisqu'il évoque les discussions des autres spectateurs pendant l'entr'acte et son attente de l'actrice à la sortie des artistes, mais son intention d'emmener l'actrice avec lui dans la forêt témoigne de la puissance du processus de cocréation entre spectateur et acteur (et non le personnage) dans l'espace théâtral évoqué par Herrmann. Il est en ce sens significatif que le menuisier n'attache aucune importance à l'autographe de l'actrice qu'il a perdue, mais raconte encore et encore son expérience. Schimmelpfennig attire l'attention sur l'ambivalence de ce récit. C'est sur la base de ce récit que la fille du menuisier et son fiancé se mettent à la recherche du bois pour le 
plancher et se transforment en arbres. Mais c'est sur la base du même récit que le bûcheron envisage non seulement de construire le théâtre, mais encore de quitter la forêt puisque le théâtre peut en ville fonctionner comme une hétérotopie de l'illusion et de compensation de la forêt. Pour le bûcheron se dessine ainsi la possibilité de se libérer de l'ordre mythique pour rejoindre l'ordre de l'emplacement, le seul, selon Foucault, à disposer d'hétérotopies.

\section{Le théâtre comme espace hétérotopique}

Dans son auto-référentialité et iconicité, Aus den Städten in die Wälder, aus den Wäldern in die Städte fait ressortir de façon aiguë une contradiction que contiennent en germe également les autres pièces évoquées. Car l'expérience du spectateur occupé à dénoter les signes $\mathrm{du}$ récit du basculement ne correspond- notamment au moment de la transformation des personnages en arbres - en rien à l'expérience d'un espace vécu, narrée par le menuisier. Le mode postépique, en ce qu'il libère la scène de la nécessité de représenter ce type de basculements, est une réponse technique à cette difficulté dont Schimmelpfennig souligne l'importance ${ }^{66}$. Néanmoins, la contradiction persiste et elle a son sens. Car la réception spatiale décrite par le menuisier et visée également par l'image de la disparition dans le tableau dans Avant/Après est en réalité elle-même avant tout déterminée par l'appartenance des personnages concernés à l'espace mythique. Or Schimmelpfennig qui dit adhérer à un théâtre "qui n'essaie pas de me tromper " [ « solange es mir nichts vormacht »] vise une forme de réception spatiale compatible avec une conscience de la médiation opérée par le théâtre. Il mène pour cela la forme narrative au seuil de la spatialité.

Dans les pièces postépiques et narrées, les effets de distanciation issus du théâtre épique, ainsi que le recours à des instances de narration de type personnage-narrateurs et narracteurs introduisent une scission importante, perceptible pour le spectateur, entre l'espace dramatique et l'espace scénique et les maintiennent dans une coexistence simultanée. Le spectateur qui s'investit dans ces deux espaces vit ainsi un dédoublement de l'espace que Schimmelpfennig utilise pour réintroduire une forme de réception spatiale en rendant l'espace théâtral instable. Il joue pour cela sur l'alternance entre la coexistence de l'espace dramatique et l'espace scénique avec des phénomènes de superposition et de court-circuitage. Les effets de superposition sont introduits par Schimmelpfennig de deux manières distinctes : par le basculement du mode diégétique dans le mode mimétique d'une part, et par la stimulation d'une réception autant spatiale que médiale d'autre part. Le basculement dans le mode mimétique est effectué notamment par l'introduction de discours directs et de parties dialoguées dans les récits faits par les personnages-narrateurs dans les pièces postépiques. Ce sont donc des moments où l'espace dramatique s'incarne dans l'espace scénique (il va sans dire que la mise en scène peut accentuer ou minimiser cet effet). Dans les pièces postépiques, ces moments de bascule sont nombreux. Dans les pièces entièrement narrées, ces moments sont plus ambivalents. A l'exception du Dragon d'or, ils sont plus fugitifs aussi. Ils reposent souvent sur de simples effets de répétition lorsque le récit d'un membre du chœur comporte un discours direct introduit ou suivi par un verbe du dire et que ce discours direct est repris ensuite par un autre membre du choeur, notamment lorsque celui-ci a le profil (sexe et âge) du personnage : « UNE FEMME AUTOUR DE LA QUANRANTAINE [...] Je reviens tout de suite, / dit une femme dans une jupe bleu foncé / à voix basse à son 
mari, / tu veux aller où, / lui chuchote-t-il UN HOMME AUTOUR DE LA QUANRANTAINE Tu veux aller où ? ${ }^{67}$ Le statut ontologique de l'espace scénique change donc ici et rend l'espace théâtral instable.

La stimulation d'une réception autant spatiale que médiale repose quant à elle chez Schimmelpfennig sur la matérialité / musicalité du récit et de la voix (des voix) qui le porte(nt). Il fait commenter ce principe par la première comédienne dans Keine Arbeit für die junge Frau im Frühlingskleid en réponse à l'objection de la deuxième comédienne : « 2e COMEDIENNE Une série écrite de sons les fixe d'une manière absolue. Pourtant, c'est justement cette fixité qui éveille en nous un univers programmé d'émotions apparentes. Cela mène parfois jusqu'à un sentiment étrange de sublime. Mais ce sentiment ne s'apparente pas encore à une pensée. Si vous êtes d'accord avec moi sur le fait qu'il y a un lien direct entre pensée et langage, alors le langage et la musique sont incompatibles, car la musique ne transporte pas le langage, mais des sons. 1ère COMEDIENNE Ce sont là des foutaises. Le dialogue ne se limite pas à l'expression langagière. Il ne l'a jamais fait. Et pourquoi devrions-nous continuer à séparer émotions et pensées! $\aleph^{68}$ Un exemple particulièrement intéressant de cette stimulation parallèle des deux modes de réception est constitué par le voyage dans l'Autre monde du jeune Chinois dans Le Dragon d'or. En rupture avec le rythme du reste de la pièce qui repose sur l'alternance des voix, ce récit très long, porté par la seule actrice qui présente le jeune Chinois, introduit un flot de parole qui entraîne le spectateur et recrée par ses propres moyens autant le courant qui porte le corps vers la Chine que la durée démesurée de ce voyage mythique. Dans les pièces entièrement narrées ce sont souvent les répétitions-variations qui créent des effets d'envoûtement. Ralentissant l'avancée du récit par des effets de narration en spirale, elles créent alors une dynamique qui est ici souvent indépendante du récit et incite le spectateur à un mode spatial de réception.

En dehors de ces effets de superposition, Schimmelpfennig introduit, souvent à la fin de ses pièces, des éléments qui créent des court-circuits entre les sphères ontologiques installées. C'est ainsi qu'à la fin d'Avant/Après, la seule didascalie de la pièce introduit dans cette pièce entièrement portée par les voix, le mouvement paradoxal d'un corps dans l'espace : un homme marche sur le mur, traverse la pièce par le plafond et redescend par le mur d'en face. Jusqu'à ce moment, le spectateur avait certes assisté à des moments fugitifs où le dialogue s'était incarné sur scène, rendant instable l'espace scénique. Les personnages-narrateurs avaient aussi introduits l'espace théorique et l'espace mythique dans leurs dialogues et récits, certains comme l'homme dans le tableau avaient basculé dans un espace autre, mais le spectateur était resté dans une sphère ontologique distincte. Ce mouvement à la fin de la pièce, qui évoque tant l'absence de l'attraction terrestre dans l'espace astronomique que la présence de lois spatiales incompatibles avec l'expérience du spectateur dans l'espace mythique, tend à faire basculer le spectateur à son tour dans un autre ordre spatial. On retrouve ce mode de fonctionnement dans Une Nuit arabe lorsque Lohmeier revient de l'orient rêvé avec un objet bien réel (encore que cela se passe dans le mode diégétique) et surtout, sur un mode plus burlesque, lorsque Schimmelpfennig termine le récit de la chute de l'homme enfermé dans la bouteille (et la pièce dans son ensemble) par la seule didascalie de la pièce introduisant « une bouteille qui tombe du plafond de la scène et se brise $»^{69}$. Dans Hier und Jetzt, le court-circuit est créé dans l'autre sens. Ici, Schimmelpfennig part d'un espace iconique décrit par une longue didascalie. Une longue tablée est installée dans la forêt et accueille un repas de mariage. Les invités se lèvent tour à tour et profèrent un récit sur un mode choral. Celui- 
ci commence par l'évocation d'un espace concurrent à l'espace mimétique et introduit ensuite le récit d'une rencontre amoureuse de la mariée avec un autre homme, puis leur relation adultère, puis la séparation du couple des mariés, le duel des deux hommes et l'errance du marié. Si cette histoire est en principe postérieure au récit incarné sur scène, cette hypothèse est invalidée par le fait que l'amant est présent à la fête de mariage et que l'espace narré dans le récit des invités s'incarne par moments sur un mode mimétique. Le récit enchâssé devenant enchâssant, Schimmelpfennig brouille les niveaux diégétiques et crée ainsi une oscillation de l'espace théâtral. En intégrant la spatialité également comme «expérience vécue » (Herrmann) du spectateur (instabilité du statut de l'espace scénique), Schimmelpfennig procure à son spectateur l'expérience de basculements, de glissements, de seuils et d'instabilités qu'il fait vivre aussi à ses personnages, à ceci près que cela se passe dans l'«espace esthétique " que Cassirer conçoit dans une tradition kantienne et schillerienne comme un espace de liberté. Le spectateur n'y subit plus «le mouvement vital immédiat qui s'exprime dans les affects mythiques fondamentaux de l'espoir et de la peur, dans l'attirance et la répulsion magiques, dans l'avidité de la saisie du "sacré" et dans l'horreur du contact avec l'interdit et le profane. Car comme contenu de la présentation artistique, l'objet s'est porté à une nouvelle distance, il s'est éloigné du Moi et ce n'est que là qu'il a acquis son être propre indépendant, une nouvelle forme de l'objectivité' $»^{70}$.

La dramaturgie de Schimmelpfennig prend tout son sens lorsque, dans certaines pièces, la tension entre narrativité et spatialité s'étend également sur la problématique abordée. C'est le cas lorsque les problèmes sociaux de l'espace mondialisé rencontrent la fonction anthropologique de la narration contenue dans les grands mythes fondateurs. L'expérience esthétique du spectateur rejoint alors non seulement le parcours des personnages, mais aussi l'ancrage thématique et générique des pièces : sur le seuil. Une belle leçon de théâtre.

\section{NOTES}

1. Theresia Birkenhauer, «Zwischen Rede und Sprache, Drama und Text: Überlegungen zur gegenwärtigen Diskussion », in : Hans-Peter Bayerdörfer (dir.), Vom Drama zum Theatertext ? Zur Situation der Dramatik in Ländern Mitteleuropas, Tübingen, Niemeyer, 2007, p. 15: "Neue Theatertexte lassen sich nicht mehr durch typologische Merkmale des Dramas klassifizieren, weder durch die Respektierung dialogischer Strukturen noch durch andere Formelemente. »

2. Ibid., p. 23.: «[...] ob ein Text ein Theatertext ist oder nicht. [...] entscheidet sich vielmehr daran, inwiefern Texte mit dem Potential theatraler Darstellungsstrukturen arbeiten. »

3. Après une formation de mise en scène (non achevée) à la Otto Falckenberg-Schule à Munich, Schimmelpfennig devient l'assistant de Dieter Dorn. Depuis la mort de son metteur en scène de prédilection, Jürgen Gosch, il met souvent en scène ses propres pièces. Pour des éléments biographiques et son rapport au théâtre voir Roland Schimmelpfennig, Ja und Nein. Vorlesungen über Dramatik. Saarbrücker Poetikdozentur für Dramatik, édité par Johannes Birgfeld, Berlin, Theater der Zeit, 2014. Pour son rapport à Jürgen Gosch, voir également les travaux de Stefan Tigges, p.e. «Der Sprung in der Scheibe, mit dem alles begann. Wort-Regie-Theater. Roland Schimmelpfennigs 
Hier und Jetzt als polyphone Raum-Zeit-Variation », in Artur Pelka, Stefan Tigges (éd.), Das Drama nach dem Drama. Verwandlungen dramatischer Formen in Deutschland seit 1945, Bielefeld, Transcript, 2011, p. 221-244.

4. Il vient toutefois de publier son premier roman An einem klaren, eiskalten Januarmorgen zu Beginn des 21. Jahrhunderts (Frankfurt / Main, Fischer, 2016).

5. Voir Roland Schimmelpfennig, « Narratives Theater », in Bernd Stegemann (éd.), Lektionen 1: Dramaturgie, Berlin, Theater der Zeit, 2009, p. 315-317.

6. Oskar Schlemmer, « Die Bauhausbühne », in Das Werk, n¹5 / 1928, Heft 1, p. 8.

7. Max Herrmann, «Das theatralische Raumerlebnis » [1931], in Jörg Dünne, Stephan Günzel (dir.), Raumtheorie. Grundlagentexte aus Philosophie und Kulturwissenschaften, Francfort/Main, Suhrkamp, 2006, p. 501-514.

8. A l'inverse de la terminologie introduite par Brecht qui parle d'un théâtre non-aristotélicien pour désigner son propre théâtre épique, Lehmann range Brecht en-deçà de la rupture postdramatique / postnarrative puisque la fable (le mythos aristotélicien) reste centrale dans sa dramaturgie. Voir Hans-Thies Lehmann, Postdramatisches Theater, Berlin, Verlag der Autoren, 1999. La thèse de la non-narrativité du théâtre post-dramatique a été à son tour contredite ces dernières années. Voir notamment Benoît Hennaut, Théâtre et récit, l'impossible rupture: narrativité et spectacle postdramatique [1975-2004], Paris, Garnier, 2016. Voir également Nina Tecklenburg, Performing Stories. Erzählen in Theater und Performance, Bielefeld, Transcript, 2014.

9. Peter Brook, L'Espace vide. Ecrits sur le théâtre, traduit de l'anglais par Christine Etienne et Franck Fayolle, Paris, Seuil, 1977, ici p. 25.

10. Eric Bentley, The life of the drama, New York, Atheneum, 1964, p. 150.

11. Il est évident en revanche que cette définition se prête particulièrement bien à définir comme théâtrales d'autres situations de représentation (juridiques, politiques, etc.).

12. Anne Ubersfeld, «Espace et théâtre » in Michel Corvin (dir.), Dictionnaire encyclopédique du théâtre, p. 506.

13. Ibid.

14. Peter Brook, 1991, p. 26.

15. Voir Michel Foucault, «Des espaces autres» (conférence au Cercle d'études architecturales, 14 mars 1967), in Architecture, Mouvement, Continuité, n5, octobre 1984, pp. 46-49, également consultable en ligne http://foucault.info/doc/documents/heterotopia/foucault-heterotopia-enhtml. Voir notamment la définition initiale : «Il y a [...] des lieux réels, des lieux effectifs, des lieux qui sont dessinés dans l'institution même de la société, et qui sont des sortes de contreemplacements, sortes d'utopies effectivement réalisées dans lesquelles [...] tous les autres emplacements réels que l'on peut trouver à l'intérieur de la culture sont à la fois représentés, contestés et inversés, des sortes de lieux qui sont hors de tous les lieux, bien que pourtant ils soient effectivement localisables. Ces lieux, parce qu'ils sont absolument autres que tous les emplacements qu'ils reflètent et dont ils parlent, je les appellerai, par opposition aux utopies, les hétérotopies [...]. »

16. Ibid.

17. Pour la distinction entre lieu et espace, voir Michel de Certeau, L'invention du quotidien, tome 1: Arts de faire, nouvelle édition, Paris, Gallimard, 1990, notamment p. 172-173 : «Est un lieu l'ordre (quel qu'il soit) selon lequel des éléments sont distribués dans des rapports de coexistence. [...] Il y a espace dès qu'on prend en considération des vecteurs de direction, des quantités de vitesse et la variable de temps. L'espace est un croisement de mobiles. [...] En somme, l'espace est un lieu pratiqué. »

18. Herrmann 1931, p. 502 : «Der Raum, den das Theater meint, ist vielmehr ein Kunstraum, der erst durch eine mehr oder weniger große innerliche Verwandlung des tatsächlichen Raumes zustande kommt, ist ein Erlebnis, bei dem der Bühnenraum in einen andersgearteten Raum verwandelt wird». 
19. Patrice Pavis, Voix et images de la scène, note 126, p. 389

20. Herrmann, 1931, p. 508 : "Jene schöpferische, mitschöpferische Tätigkeit des Publikums an "allem" schauspielerischen Spiel besteht zu allertiefst in einem heimlichen Nacherleben, in einer schattenhaften Nachbildung der schauspielerischen Leistung, in einer Aufnahme nicht sowohl durch den Gesichtssinn wie vielmehr durch das Körpergefühl, in einem geheimen Drang, die gleichen Bewegungen auszuführen, den gleichen Stimmklang in der Kehle hervorzubringen. »

21. Ibid., p. 505 / 506 : «Jeder Mensch ist von dem Raum, in dem er sich jeweilig befindet, in bezug auf seinen Habitus durchaus abhängig : unser Gehen, unsere Gesten, unser Sprechen sind anders in der freien Natur als im geschlossenen Raum und im einzelnen wieder entscheidend von den Besonderheiten dieser Natur oder dieses geschlossenen Raumes bedingt. [...] der bedeutende Schauspieler schafft sich seinen Raum selber oder, genauer, er deutet sich den Bühnenraum um in einen tatsächlich nicht vorhandenen Realitätsraum, der nun seinen ganzen Habitus in dem vorhin gekennzeichneten Sinne bedingt. »

22. Monika Fludernik, Towards a «natural » narratology, Londres \& New York, Routledge, 1996, p. 333. Traduction Kerstin Hausbei.

23. Benoît Hennaut, " Narratologie et écritures théâtrales : quel dialogue possible? », Cahiers de Narratologie [en ligne], 24 | 2013, URL : http://narratologie.revues.org/6669 [consulté le 25 juin 2016], p. 2.

24. Pour l'histoire des concepts, notamment chez Platon et Aristote, voir Stephen Halliwell, «Diegesis - Mimesis », in Peter Hühn et al. (éd.), the living handbook of narratology. Hambourg, Hamburg University, URL http://www.lhn.uni-hamburg.de/article/diegesis---mimesis [consulté le 26 juin 2016]. Pour l'histoire de leur utilisation dans la constitution théorique des genres et la construction d'une théorie narrative avant 1900, voir Matthias Grüne, « Das vergessene Erbe. Zur Konzeption einer Geschichte der Erzähltheorie", DIEGESIS. Interdisziplinäres E-Journal für Erzählforschung/Interdisciplinary E-Journal for Narrative Research 3.2 / 2014, p. 50-65. URL : http:// www.diegesis.uni-wuppertal.de/index.php/diegesis/article/download/167/225 [consulté le 26 juin 2016].

25. Pour un résumé du fondement théorique de l'exclusion du théâtre et du drame du champ de la narratologie, puis de leur réintégration toujours en cours, voir Benoît Hennaut 2013.

26. Voir par exemple Arielle Meyer MacLeod, Michèle Pralong (dir.), Raconter des histoires. Quelle narration au théâtre aujourd'hui ?, Genève, Metis Press, 2012.

27. Voir Seymour Chatman, Coming to terms: the rhetoric of narrative in fiction and films, Ithaca, Cornell UP, 1990, notamment p. 109-118.

28. Des espaces de jeu et de projection multiples permettant de créer des montages de documents hétérogènes que la mise en scène invite le spectateur à mettre en rapport avec la narration.

29. Je retiens cette notion introduite par Hans-Thies Lehmann plutôt que celle, alternative, de «théâtre narratif» («Erzähltheater») qui prête plus à confusion (voir infra le passage sur le théâtre postépique).

30. Voir à ce sujet Didier Plassard, "Le Postdramatique, c'est-à-dire l'abstraction ", Prospero European Review. Theatre and research, 3/2012, URL : http://www.t-n-b.fr/en/prospero/europeanreview/fiche.php?id=91\&edition=10\&lang=1. Voir également Hans-Thies Lehman, op. cit., où il définit le théâtre postdramatique comme un théâtre postbrechtien en arguant du fait que Brecht n'aurait pas aboli la fable comme élément central de la représentation théâtrale alors que le théâtre postdramatique aurait précisément franchi ce pas décisif.

31. Bernd Stegemann, "Dramaturgie des Postdramatischen», in idem (éd.), Lektionen 1: Dramaturgie, Berlin, Theater der Zeit, 2009, p. 287-288 : «Die Kunst der Parataxe besteht darin, dieses Nebeneinander so zu gestalten, dass die Wirkung auf der Bedeutungsebene offen und auf der Gefühlsebene dennoch affizierend ist. In der dramatischen Abfolge besteht zwischen dem Erkennen und der emotionalen Wirkung eine dialektische Beziehung. In der parataktischen 
Abfolge fehlt der erkenntnisleitende rote Faden einer Handlung. Der Zuschauer muss in jedem Moment selbst zur sinnlichen Anteilnahme bereit sein. Erst wenn er diese zulässt und erlebt, kann er den Ablauf der Parataxen als künstlerische Form erfahren. [...] Er wird zum Autor der von ihm selbst erlebten Ereignisse. Dazu muss er diese jedoch zuvor erlebt haben und darf sich diese Erleben nicht durch eine den Sinn suchende Beobachtung verstellen. »

32. Le concept, introduit par Volker Klotz, recouvre dans les grandes lignes les caractéristiques de ce que Peter Szondi a appelé le drame absolu, soulignant ainsi qu'il s'agit d'un idéal rarement - jamais ? - réalisé dans l'écriture dramatique. Voir Volker Klotz, Formes ouvertes et formes fermées dans le drame (1960), traduction par Claude Maillard, Paris, Circé, 1994.

33. La notion de didascale a été introduite par l'hispaniste Monique Martinez Thomas (voir « Le didascale dans l'histoire du théâtre espagnol ", in Sandra Golopentia et Monique Martinez Thomas Voir les didascalies, Toulouse/Paris, Cric \& Ophrys, 1994, p. 135-231). Elle désigne l'instance énonciative des didascalies qui organise aussi la distribution de la parole et doit être tenu pour responsable de la parole des personnages. Si Anne Uberfeld identifie cette instance (qu'elle appelle " énonciateur-scripteur ») encore largement à l'auteur (voir Lire le théâtre III. Le dialogue de théâtre, Paris, Belin, 1996, p. 53-57), on considère aujourd'hui souvent qu'il s'agit d'une instance analogue au narrateur dans le roman (voir p.e. Éric Eigenmann qui transpose la narratologie de Gérard Genette dans l'analyse dramatique : "Méthodes et problèmes : Le mode dramatique ", 2003, document en ligne, URL https://www.unige.ch/lettres/framo/ enseignements/methodes/modedramatique/mdintegr.html\#md014200 [dernière consultation le 12.1.2017]).

34. Voir par exemple Holger Korthals, Zwischen Drama und Erzählung. Ein Beitrag zur Theorie geschehensdarstellender Literatur, Berlin, Erich Schmidt-Verlag, 2003.

35. Il n'est en ce sens pas logique que Schmid réintroduise la présence d'un narrateur comme critère pour distinguer entre des «textes narratifs médiatisés" et des «textes narratifs mimétiques » (parmi lesquels il compte non seulement le théâtre, mais explicitement le drame) dans lesquels "le changement de situation est représenté sans instance de médiation ». Voir Wolf Schmid, Elemente der Narratologie, Berlin / Boston, De Gruyter, 2014, p. 8-9.

36. Voir Wolf Schmid, «La constitution narrative : les événements - l'histoire - le récit - la représentation du récit ", in John Pier (dir.), Théorie du récit. L'apport de la recherche allemande, Villeneuve d'Ascq, Septentrion, 2007, p. 153-188.

37. Voir Chatman 1990. Dès qu'on quitte le paradigme du «drame absolu» tel que défini par Peter Szondi en se référant aux écritures dramatiques et conditions de mise en scène d'une période historique bien déterminée, il faut de toute façon introduire l'idée d'une instance épique (voir sa Théorie du drame moderne [1956], traduction de Sibylle Muller, Paris, Circé, 2006).

38. Korthals 2003, p. 57: «[...] der illusionierte Zuschauer, der sich über den Vermittlungsrahmen der Bühne, der Schauspieler und des theatralen Apparats hinwegsetzt und das Geschehen gleichsam miterlebt, seinerseits als eine Illusion bestimmter Vertreter der Dramentheorie angesehen werden kann. »

39. On pense bien sûr à l'esthétique du théâtre épique telle qu'elle a été théorisée dans les Ecrits sur le théâtre de Brecht. Voir également Theresia Birkenhauer, 2007, qui insiste sur l'utilisation de la langue dans les textes et mises en scène, ainsi qu'Anne Bédard qui développe l'idée d'un dialogisme du théâtre qu'elle prête aux formes «expérimentales » de la mise en scène: «Du discours de l'espace théâtral. Du monologisme au dialogisme", L'Annuaire théâtral: revue québécoise d'études théâtrales, nº 7 / 1990, p. 83-92.

40. Hans-Peter Bayerdörfer, « Zurück zu großen Texten ? Dramaturgie im heutigen Erzähltheater ", in : Artur Pelka, Stefan Tigges (éd.), Das Drama nach dem Drama. Verwandlungen dramatischer Formen in Deutschland seit 1945, Bielefeld, Transcript, 2011, p. 168 : «Der Schauspieler agiert als Geschehensteilnehmer im interpersonalen Geschehen, aber latent immer zugleich körperlich als Geschehensvermittler, d.h. als körperlich erzählender Interpret seiner Verkörperung. » 
41. Voir par exemple les travaux de Ansgar Nünning et Roy Sommer.

42. Voir par exemple Tecklenburg, 2014, ainsi que Simone Soriani, «Théâtre de narration / théâtres de la narration : Marco Paoliniet Ascanio Celestini », Chroniques italiennes 27 (1/2014), 160-176.

43. Soriani propose le concept du «théâtre de la narration " pour des spectacles fonctionnant entièrement sur ce mode qui privilégie l'axe scène-salle au détriment de la double communication que l'on prête habituellement au texte dramatique.

44. Voir Walter Benjamin, «Expérience et pauvreté » et «Le conteur ». Voir également JeanneMarie Gagnebin, Histoire et Narration chez Walter Benjamin, Paris, L'Harmattan, 1991.

45. Voir Hennaut 2011, p. 12: «par l'étude de textes critiques produits à la réception des spectacles, on peut envisager la mobilité du discours narratif organisateur du sens du spectacle en dehors de l'objet spectaculaire lui-même, le situer dans la rencontre des éléments portés par la performance et du langage articulé par un critique ou un spectateur qui s'en saisit. » On peut toutefois se demander si le discours organisateur de sens est alors (forcément) de type narratif.

46. Sur les mises en scène de romans - encore très en vogue actuellement - voir à côté de HansPeter Bayerdörfer, 2011, l'article précoce de Hajo Kurzenberger dans lequel il évoque également l'importance de Peter Brook pour l'apparition d'un théâtre narratif: «Erzähltheater. Zur Theatralisierung epischer Texte von Franz Kafka und Marguérite Duras », in Erika Fischer-Lichte, Wolfgang Greisenegger, Hans-Thies Lehmann (éd.), Arbeitsfelder der Theaterwissenschaft, Tübingen, Narr, 1994, p. 171-182.

47. Pour ce concept, voir Stegemann 2009, p. 304-311.

48. Voir par exemple Hans-Peter Bayerdörfer (dir.), Vom Drama zum Theatertext? Zur Situation der Dramatik in Ländern Mitteleuropas, Tübingen, Niemeyer, 2007; Anne Monfort, "Après le postdramatique : narration et fiction entre écriture de plateau et théâtre néo-dramatique ", Trajectoires [en ligne] 3 / 2009 : Mondes en narration, mis en ligne le 16 décembre 2009, URL : http://trajectoires.revues.org/392.

49. Voir Schimmelpfennig 2014, p. 20.

50. Franz Wille, «Eine aufregende Zeit um für das Theater zu schreiben. Ein Gespräch mit Roland Schimmelpfennig ", Theaterheute, Jahrbuch 2010, p. 119.

51. Voir à ce sujet Erika Fischer-Lichte, "Aufführung ", in idem, Doris Kollesch, Matthias Warstat (dir.), Metzler Lexikon Theatertheorie, Stuttgart / Weimar, Metzler, 2005, p. 16-26.

52. Pour cet aspect, voir la thèse de Christine Laudahn qui contient également l'interprétation sémantique de certaines mises en scène: Zwischen Postdramatik und Dramatik. Roland Schimmelpfennigs Raumentwürfe, Tübingen, Narr Francke Attempto Verlag, 2012.

53. D'abord chantée par une «femme au balai » dans la sphère visuelle, elle est reprise en partie par LUI dans la sphère du dialogue, avant de rebasculer dans la sphère visuelle où elle est reprise par deux autres « femmes au balai ».

54. Par exemple : «LUI Tu te souviens ? / ELLE Mais oui, bien sûr. Comment pourrais-je ne pas me souvenir ? [...]» ou encore « ELLE Et le vélo ? / LUI Ah oui, le vélo... / ELLE Tu ne veux pas en parler du vélo, n'est-ce pas? Alors dis-moi comment tu vas / LUI Je vais bien. / ELLE Oui ? / LUI Oui, je vais bien. Et toi ? Qu'est-ce que tu deviens ? Comment vas-tu? », Schimmelpfennig 2004, respectivement p. 138 et p. 140 : «ER Erinnerst du dich? / SIE Aber ja, natürlich. Wie könnte ich mich nicht erinnern?»; «Sie Was ist mit dem Fahrrad ? / ER Ach, das Fahrrad... / SIE Du willst nicht über das Fahrrad sprechen, oder ? Dann sag mir, wie es dir geht. / ER Es geht mir gut. / SIE Ja ? / ER Ja, es geht mir gut. Und du ? Was machst du ? Wie geht es dir?»

55. Par exemple : «LUI Il y avait quoi dans cette valise? / ELLE Tu ne voulais pas arrêter de me poser des questions? » ou encore « ELLE Tu l'as toujours le vélo? Toi ? / LUI Le vélo ? Ah oui, le vélo - pourquoi tu poses cette question?», ibid., respectivement p. 144 et p. 147 : «ER Was war in dem Koffer ? / SIE Wolltest du nicht aufhören zu fragen? » / « SIE Hast du eigentlich das Fahrrad noch ? Du ? / ER Das Fahrrad ? Ja, das Fahrrad - warum fragst du?» 
56. Par exemple : «ELLE Tu as dit que tu l'avais toujours, le vieux vélo? / LUI Oui, oui. Je pense que oui, je crois bien - je ne sais pas » ou encore «LUI Et ton amie ? Comment va-t-elle ? / ELLE Je ne sais pas. ", ibid., respectivement p. 136 et p. 138 : «SIE Du hast das alte Fahrrad noch, hast du gesagt. / ER Ja, ja. Ich denke doch, ich glaube schon - ich weiß nicht.»/ «ER Und deine Freundin? Wie geht es ihr? / SIE Ich weiß nicht. »

57. Voir Marie-Laure Ryan, «Space », in the living handbook of narratology [consulté le 17.7. 2016].

58. Push-Up, traduction d'Alexis-Henri Baatsch, Paris, Arche, 2002, p. 83. La traduction a été légèrement modifiée pour faire ressortir que le personnage s'est réellement rendu dans ces pays asiatiques et n'a pas seulement travaillé pour des sociétés basées dans ces pays. Cette nuance, importante dans notre contexte, ressort clairement du texte allemand: "Sie haben in Amerika den Abschluß gemacht und waren anschließend für zwei Firmen in Japan, Korea und Taiwan ", Schimmelpfennig 2004, p. 349.

59. Push Up, p. 77.

60. Le Dragon d'Or, traduction de Hélène Mauler et René Zahnd, Paris, Arche, 2011, p. 41.

61. Ernst Cassirer, «Espace mythique, espace esthétique et espace théorique » [1930], traduction Christian Berner, in idem, Ecrits sur l'art, éd. par Fabien Capeillères, Paris, Cerf, 1995, p. 109.

62. Ibid.

63. Foucault 1984.

64. Voir Schimmelpfennig 2004, P. 232-233 : «ERNST Jamais, mon vieil ami, je ne t'ai demandé de causer notre perte, la tienne, la mienne et celle de nos bien-aimés, de nous précipiter dans la chute certaine, de nous livrer à la terrible jalousie de cette forêt que, une fois déclenchée, personne ne pourra calmer ! [...] Ce n'est pas une chose utile! Pour cela, la forêt de hait avec ses arbres que je suis sensé abattre pour toi - que tu leur prend leur habitat sans la moindre raison. » / «ERNST Niemals, mein alter Freund, habe ich dich darum gebeten, uns, dich wie mich und unsere Lieben, ins Verderben, in den sicheren Untergang $\mathrm{zu}$ stoßen, der furchtbaren Eifersucht dieses Waldes auszuliefern, die niemand, einmal erste entfacht, zu besänftigen vermag ! [...] Das ist kein Nutzen! Dafür haßt dich der Wald mit seinen Bäumen, die ich für dich schlagen soll - daß du die Wohnung ihnen nimmst ohne den geringsten Sinn. »

65. Lotman défend l'idée selon laquelle toute histoire repose sur une topologie binaire dont les parties sont séparées par une frontière, franchissable par le seul héros de l'histoire. Lorsque cette frontière entre les deux espaces sémantiques est effectivement franchie, Lotman considère que l'histoire est « à sujet ».

66. Voir Schimmelpfennig 2009.

67. Schimmelpfennig 2011, p. 652 : «EINE FRAU UM DIE VIERZIG [...] Ich bin gleich wieder da, / sagt eine Frau in einem dunkelblauen Rock / leise zu ihrem Mann, / wo willst du hin, / flüstert er / EIN MANN UM DIE VIERZIG Wo willst du hin?»

68. Schimmelpfennig 2004, p. 119 : «2. SCHAUSPIELERIN Eine notierte Reihe von Tönen ist eine vollkommene Festlegung. Dennoch ist es genau diese Festlegung, die in uns eine abrufbare Welt von scheinbaren Gefühlen zum Leben erweckt. Das führt bisweilen zu einem sonderbaren Gefühl der Erhabenheit. Mit einem Denkvorgang hat dieses Gefühl aber noch nichts zu tun. Wenn ihr mir recht gebt, dass Gedanken und Sprache einen unmittelbaren Zusammenhang haben, so sind Sprache und Musik unvereinbar, denn Musik trägt nicht Sprache, sondern Töne. / 1 . SCHAUSPIELERIN Das ist alles Unsinn. Der Dialog ist längst nicht nur eine Form des sprachlichen Ausdrucks. Das ist er auch nie gewesen. Und warum sollen wir länger Gefühle und Gedanken trennen!»

69. Roland Schimmelpfennig, Une Nuit arabe, traduit de l'allemand par Johannes Honigmann avec la collaboration de Laurent Muhleisen, in idem, Une nuit arabe. Push Up, Paris, Arche, 2002, p. 74.

70. Cassirer 1995, p. 112. 


\section{ABSTRACTS}

Le théâtre est autant un art de l'espace qu'un vecteur de narration. En s'appuyant sur des concepts issus des études théâtrales, de la narratologie, de la sociologie et de la phénoménologie, l'auteure explique les enjeux et variantes de ces définitions du théâtre qui tendent à se contredire. Elle montre comment la redéfinition des concepts tout autant que la différence entre les formes dramatiques, épiques, postdramatiques et postépiques du théâtre fait bouger les lignes de ce champ de tension. Partant de l'observation que l'appartenance des textes contemporains au théâtre repose avant tout sur la façon dont ils se servent des structures de représentation de celui-ci, l'auteur se demande ensuite comment cette exigence se reflète dans la dramaturgie éminemment narrative de Roland Schimmelpfennig. Sur la base des différentes catégories de narrativité, elle introduit une classification des formes dramaturgiques utilisées et montre comment les aspects parataxiques et hétérotopiques de la spatialité sont utilisés pour créer des effets de seuils et de liminalité qui plongent le spectateur dans une expérience esthétique autour de la spatialité, lui permettant de prendre conscience de ce qu'est ou peut être l'espace.

Das Theater ist gleichzeitig Raumkunst und Medium des Erzählens. Unter Bezugnahme auf Konzepte aus Theaterwissenschaft, Narratologie, Soziologie und Phänomenologie erklärt die Autorin die Implikationen und Ausprägungen dieser einander widerstrebenden Definitionen des Theaters und verfolgt, wie sich die Reibungsflächen zwischen beiden Definitionen einerseits durch die Umdeutung der Konzepte, andererseits durch die dramatische, epische, postdramatische oder postepische Ausrichtung des Theaters verschieben. Ausgehend von der Beobachtung, dass sich zeitgenössische Theatertexte nur dadurch dem Theater zuordnen lassen, dass sie dessen Darstellungsstrukturen nutzen, fragt sich die Autorin in einem zweiten Schritt, wie sich das in der dominant narrativen Dramaturgie Roland Schimmelpfennigs gestaltet. Dazu ordnet sie zunächst die Stücke aller Schaffenphasen den verschiedenen Kategorien von Narrativität zu und zeigt dann, wie in ihnen die parataktischen und heterotopischen Aspekte von Räumlichkeit dazu genutzt werden, dem Zuschauer durch Liminalitäts- und Schwelleneffekte ästhetisch erfahrbar zu machen, was Raum ist und sein kann.

\section{INDEX}

Geographical index: Allemagne

Mots-clés: Schimmelpfennig, dramaturgie, postépique, hétérotopie, théâtre narratif

Chronological index: 1995-2015

\section{AUTHOR}

\section{KERSTIN HAUSBEI}

Kerstin Hausbei a reçu une double formation en études germaniques et études théâtrales et a été active dans la dramaturgie et mise en scène. Elle est maître de conférences au département d'études germaniques de l'université Sorbonne Nouvelle - Paris 3 et membre du CEREG. Cet 
article s'inscrit dans ses recherches sur la poétique des formes dramaturgiques et théâtrales. Exemples de publications dans ce domaine : «Bild, Geste und Theatralität in Franz Grillparzers Vließ-Trilogie ", in Marc Lacheny, Jacques Lajarrige et Éric Leroy du Cardonnoy (ed.), Modernité du mythe et violence de l'altérité : La Toison d'or de Franz Grillparzer, Rouen, Presses Universitaires de Rouen et du Havre, 2016 ; « Emblème interactif du commerce identitaire : Bitte liebt Österreich de Christoph Schlingensief ", Revue d'Allemagne et des Pays de Langue Allemande, vol. 45 issue 2, 2013 ; « Nestroy en version muette : Le mimodrame Les trois perruques de Marcel Marceau en 1953 à la Comédie des Champs-Elysées », Austriaca 75, 2013. 